ARTICLE

\title{
Zika virus infection induces RNAi-mediated antiviral immunity in human neural progenitors and brain organoids
}

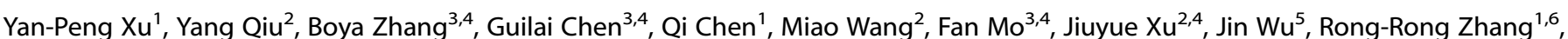
Meng-Li Cheng ${ }^{1,6}$, Na-Na Zhang ${ }^{1}$, Bao Lyu ${ }^{2,7}$, Wen-Liang Zhu ${ }^{3,4}$, Meng-Hua Wu ${ }^{3,4}$, Qing Ye ${ }^{1}$, Da Zhang ${ }^{3,4}$, Jiang-Hong Man ${ }^{5}$, Xiao-Feng $\mathrm{Li}^{1}$, Jie Cui ${ }^{2,4}$, Zhiheng Xu (iD) ${ }^{4,8}$, Baoyang Hu (iD ${ }^{3,4}, \mathrm{Xi}_{\text {Zhou }}{ }^{2,4,7}$ and Cheng-Feng Qin (iD) ${ }^{1,6}$

The re-emergence of Zika virus (ZIKV) in the Western Hemisphere has resulted in global public health crisis since 2015. ZIKV preferentially infects and targets human neural progenitor cells (hNPCs) and causes fetal microcephaly upon maternal infection. hNPCs not only play critical roles during fetal brain development, but also persist in adult brain throughout life. Yet the mechanism of innate antiviral immunity in hNPCs remains largely unknown. Here, we show that ZIKV infection triggers the abundant production of virus-derived small interfering RNAs in hNPCs, but not in the more differentiated progenies or somatic cells. Ablation of key RNAi machinery components significantly enhances ZIKV replication in hNPCs. Furthermore, enoxacin, a broad-spectrum antibiotic that is known as an RNAi enhancer, exerts potent anti-ZIKV activity in hNPCs and other RNAi-competent cells. Strikingly, enoxacin treatment completely prevents ZIKV infection and circumvents ZIKV-induced microcephalic phenotypes in brain organoid models that recapitulate human fetal brain development. Our findings highlight the physiological importance of RNAi-mediated antiviral immunity during the early stage of human brain development, uncovering a novel strategy to combat human congenital viral infections through enhancing RNAi.

Cell Research (2019) 29:265-273; https://doi.org/10.1038/s41422-019-0152-9

\section{INTRODUCTION}

Zika virus (ZIKV) is a positive-sense single-stranded RNA virus that belongs to the genus Flavivirus in the family Flaviviridae. The Flavivirus genus includes more than 50 arthropod-borne viruses with public health importance including Dengue virus (DENV), West Nile virus (WNV), Japanese encephalitis virus (JEV) and Yellow fever virus (YFV). ZIKV was firstly isolated from a sentinel monkey in the Zika forest of Uganda in $1947,{ }^{1}$ thereafter human infections with ZIKV were sporadically reported in a few African and Asian countries with mild symptoms. ${ }^{2}$ Since 2015, ZIKV unexpectedly emerged as a global public health threat because of its explosive outbreaks in the Americas and the causal link to fetal microcephaly and congenital Zika syndrome (CZS). In the following years, ZIKV continues to spread to 86 countries or territories worldwide, and it is estimated that $\sim 3.6$ billion people are now living in areas at risk for transmission. ${ }^{3}$ Despite intensive global efforts have been made to understand ZIKV pathogenesis and to develop countermeasures, no approved vaccines or antiviral drugs are currently available. ${ }^{4}$

One of the most unusual features that distinguishes ZIKV from other flavivirus members is the capability to cause devastating fetal microcephaly in infants born from infected pregnant women. ${ }^{5}$ Thousands of infants born from ZIKV-infected mothers in the Americas exhibited thinner cortical layers, the hallmark of microcephaly. 6,7 Human neural progenitor cells (hNPCs), which give rise to building blocks of human cortex, are readily identified as the major target cells of ZIKV by using cell cultures, brain organoids and fetal brain slices. ${ }^{8-13}$ More importantly, ZIKV exhibits specific tropism to hNPCs, whereas the more differentiated immature or mature neurons are less susceptible to ZIKV infection. ${ }^{8,14,15}$ ZIKV infection readily impairs hNPC proliferation and differentiation, triggers massive cell death of their progenies, and finally leads to severe brain developmental disorders, including microcephaly. ${ }^{16,17} \mathrm{~A}$ recent clinical investigation also showed that the intrinsic susceptibility of hNPCs is critical for the clinical outcome upon ZIKV infection. ${ }^{18}$ Thus, hNPCs are now widely used to study ZIKV pathogenesis and to screen and evaluate potential antiviral drugs. ${ }^{19,20}$

Innate immunity serves as the first line of host defense against invading microorganisms. In most mammalian cells, interferon (IFN) response is a major antiviral immune mechanism, which is triggered by viral infection and results in the transcriptional induction of hundreds of IFN-stimulated genes (ISGs). However, unlike most differentiated somatic cells, embryonic stem cells

\footnotetext{
${ }^{1}$ State Key Laboratory of Pathogen and Biosecurity, Beijing Institute of Microbiology and Epidemiology, Academy of Military Medical Sciences, 100071 Beijing, China; ${ }^{2}$ State Key Laboratory of Virology, Wuhan Institute of Virology, Chinese Academy of Sciences (CAS), Wuhan 430071 Hubei, China; ${ }^{3}$ State Key Laboratory of Stem Cell and Reproductive Biology, Institute of Zoology, CAS, 100101 Beijing, China; ${ }^{4}$ University of Chinese Academy of Sciences, 100049 Beijing, China; ${ }^{5}$ State Key Laboratory of Proteomics, Institute of Basic Medical Sciences, National Center of Biomedical Analysis, 100850 Beijing, China; ${ }^{6}$ School of Basic Sciences, Anhui Medical University, Hefei 230032 Anhui, China; ${ }^{7}$ College of

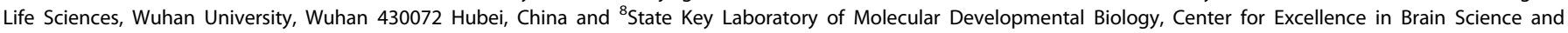
Intelligence Technology, Institute of Genetics and Developmental Biology, CAS, 100101 Beijing, China

Correspondence: Baoyang Hu (byhu@ioz.ac.cn) or Xi Zhou (zhouxi@wh.iov.cn) or Cheng-Feng Qin (qincf@bmi.ac.cn)

These authors contributed equally: Yan-Peng Xu, Yang Qiu, Boya Zhang, Guilai Chen
}

Received: 4 December 2018 Accepted: 29 January 2019

Published online: 27 February 2019 
(ESCs) and many of tissue stem or progenitor cells do not rely on the canonical IFN pathway for antiviral defense. ${ }^{21,22}$ Similarly, ZIKV infection only induced weak or delayed IFN response in hNPCs. ${ }^{23,24}$ Thus far, the mechanism by which hNPCs defend against viral infection, particularly ZIKV, remains not fully understood.

RNAi is an evolutionarily conserved post-transcriptional gene silencing mechanism in eukaryotes, which can act as an innate antiviral immune response in fungi, plants, invertebrates, and mammals. ${ }^{25,26}$ In the process of antiviral RNAi, viral replicative dsRNA intermediates generated during viral RNA replication are sensed and cleaved by host Dicer endoribonuclease into virusderived small interfering RNAs (vsiRNAs). These vsiRNAs are then loaded onto the Argonaute protein (AGO) of the RNA-induced silencing complexes (RISCs) to initiate the cleavage of cognate viral RNAs in infected cells. Though RNAi has been proposed as an antiviral immunity mechanim in mammals for a long time, ${ }^{27-30}$ the physiological importance of RNAi in mammals remains under debate. ${ }^{31-34}$ Specifically, deep sequencing of small RNA populations in mammalian cells infected with a variety of wild-type pathogenic viruses, including influenza $A$ virus (IAV), human enterovirus 71 (EV71), and Sindbis virus, has failed to detect a significant level of vsiRNAs. ${ }^{35-38}$ In addition, genetic ablation of Dicer or AGO2 failed to enhance virus replication in mammalian somatic cells. ${ }^{37,39,40}$ ZIKV can replicate in hNPCs and other somatic cells as well as mosquito cells, which provides a unique opportunity to compare RNAi-mediated antiviral immunity between human and mosquitoes. In the present study, we first detect abundant production of vsiRNAs in ZIKV-infected hNPCs with a similar potency to in mosquito cells, and ablation of key RNAi machinery components significantly enhance ZIKV replication in hNPCs. Furthermore, we demonstrate that a well-known RNAi enhancer, enoxacin, has a direct antiviral activity in RNAicompetent cells, and enoxacin treatment can completely prevent ZIKV-induced microcephalic phenotypes in brain organoids. Our results provide compelling evidence that RNAi-mediated immunity plays critical roles in defending ZIKV infection at the early stage of human brain development.

\section{RESULTS}

ZIKV infection triggers abundant production of vsiRNAs in hNPCs The production of abundant vsiRNAs within infected cells has been regarded as a hallmark of antiviral RNAi response. ${ }^{31,41}$ Here, we first sought to determine the production of vsiRNAs in ZIKVinfected hNPCs using the mosquito Aag 2 cells as a reference. The hNPCs differentiated from $\mathrm{H} 9$ human ESCs, as well as Aag2 cells, were infected with ZIKV at an MOI of 2 and total RNAs were subjected to deep sequencing for small RNA populations. Similar to previous findings, ${ }^{42,43}$ ZIKV infection in mosquito Aag2 cells readily induced abundant production of vsiRNAs that were predominantly $21-n t$ in size with $2-n t 3^{\prime}$ overhangs (Supplementary information, Fig. S1a, b) and mapped throughout the genome of ZIKV (Supplementary information, Fig. S1c). As expected, hNPCs were highly susceptible to ZIKV, featured with robust viral genome replication and NS1 protein secretion (Supplementary information, Fig. S2a-d). Notably, abundant virus-derived small RNAs (vsRNAs), 18-28 nt in length, were readily detected in ZIKV-infected hNPCs, and $62.97 \%$ of the vsRNA reads were in the size range of $21-23 \mathrm{nt}$, with 22-nt as the predominant size for both positive and negative strands (Fig. 1a and Supplementary information, Table S1). The vsRNA population was enriched for the 22-nt vsRNAs that contained a 20 -nt perfectly base-paired duplex region with 2-nt $3^{\prime}$ overhangs (Fig. $1 \mathrm{~b}$, peak " -2 "), confirming the properties of canonical siRNAs. These results show that a significant amount of vsiRNAs were produced in ZIKV-infected hNPCs.

Bioinformatic analyses of the ZIKV-specific vsRNA reads that correspond to the vsiRNA duplexes $(22 \pm 1 \mathrm{nt})$ showed that these
hNPC-derived vsiRNAs were mapped along the whole ZIKV genome, with more reads concentrated at the $5^{\prime}$-untranslated region (Fig. 1c). The vsiRNA patterns in hNPCs were similar to those detected in mosquito Aag2 cells (Supplementary information, Fig. S1). The ZIKV-derived vsRNAs detected in hNPCs formed successive complementary pairs of vsiRNAs (Fig. 1d), consistent with the characteristics of siRNAs generated by processive cleavage by Dicer ${ }^{41}$ during viral RNA replication. Moreover, ZIKVderived vsiRNAs could be detected by northern blot analysis using an RNA oligo probe complementary to nucleotides 1-50 of the ZIKV anti-genome (Fig. 1e). Together, these results show that, as in mosquito cells, the RNAi machinery in hNPCs is fully functional to cleave the dsRNAs formed during ZIKV genome replication, resulting in the production of abundant vsiRNAs.

Previous studies failed to detect any ZIKV-specific vsiRNAs in astrocytes, $^{44}$ therefore we tested whether human neurons differentiated from hNPCs retained the capability of cleaving viral dsRNA into vsiRNAs. vsRNAs detected in ZIKV-infected neurons were predominantly nonspecific viral RNA degradation products, with a random size distribution and an overwhelming bias for positive strands (Supplementary information, Fig. S3a). The lack of $22 \pm 1$-nt vsiRNA pairs in ZIKV-infected neurons suggested that the intrinsic capability of vsiRNA biogenesis decayed during the differentiation of hNPCs into neurons. In addition, we also failed to detect vsiRNAs in ZIKV-infected human rhabdomyoma RD cells (Supplementary information, Fig. S3b). These results suggest that hNPCs may represent a unique cell population in which viral dsRNAs can be efficiently processed into vsiRNAs by the RNAi machinery.

Attenuation of RNAi pathway enhances ZIKV replication in hNPCs Further, we examined the potential impact on ZIKV infection by ablation of the RNAi pathway in hNPCs. We firstly knocked down Dicer or AGO2, the two essential components of RNAi pathways, in hNPCs using two specific sets of synthesized siRNAs (Supplementary information, Fig. S4a). Interestingly, transient downregulation of either Dicer or AGO2 significantly enhanced ZIKV replication in hNPCs (Fig. 2a). We further knocked out (KO) the DCR1 gene of hNPCs by CRISPR/Cas9 system to generate two stable Dicer KO hNPCs (Supplementary information, Fig. S4b-d). Upon ZIKV infection, ZIKV-derived vsiRNAs were not detected by northern blot assay in both Dicer KO hNPCs in comparison to the wild-type hNPCs (Fig. 2b). Furthermore, ectopic expression of the Nodamura virus (NoV)-encoded B2 protein (Supplementary information, Fig. S4e), a potent viral suppressor of RNAi (VSR), ${ }^{45,46}$ significantly enhanced ZIKV replication in hNPCs, whereas the VSR-defective mutant of NoV B2 (termed "mB2") failed to do so (Fig. 2c). Thus, ablation of the RNAi pathway in hNPCs has a favorable effect on ZIKV replication. More importantly, siRNAs synthesized according to the vsiRNAs detected in the ZIKV-infected hNPCs could directly inhibit ZIKV replication in hNPCs (Fig. 2d). Besides, we also analyzed the innate IFN response in hNPCs upon ZIKV infection using human somatic A549 cells as a reference. ISG transcription and cytokine production were remarkably upregulated in ZIKVinfected A549 cells, but not in ZIKV-infected hNPCs (Supplementary information, Fig. S5a, b), suggesting that there is unique antiviral immunity in hNPCs. Taken together, these results illustrate that RNAi plays an authentic antiviral role during ZIKV infection in hNPCs.

Enoxacin exerts anti-ZIKV activity in hNPCs and brain organoids The demonstration of RNAi-mediated antiviral immunity in hNPCs highlights the possibility to develop novel antiviral strategy. Therefore, we sought to prevent ZIKV infection by manipulating the intrinsic RNAi pathway in hNPCs. Enoxacin, a clinically approved broad-spectrum antibiotic, has been well characterized as a potent RNAi enhancer. ${ }^{47,48}$ The effects of enoxacin on the $\mathrm{RNAi}$ response was validated using EGFP-expressing 
a

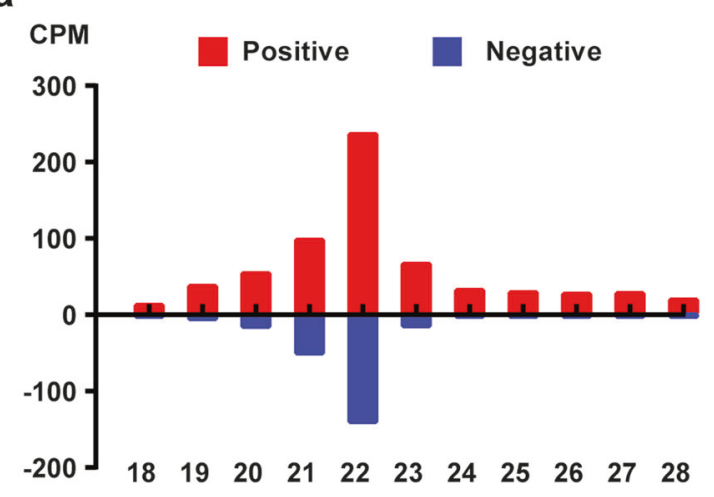

Reads Length in Nucleotides (nt)

C

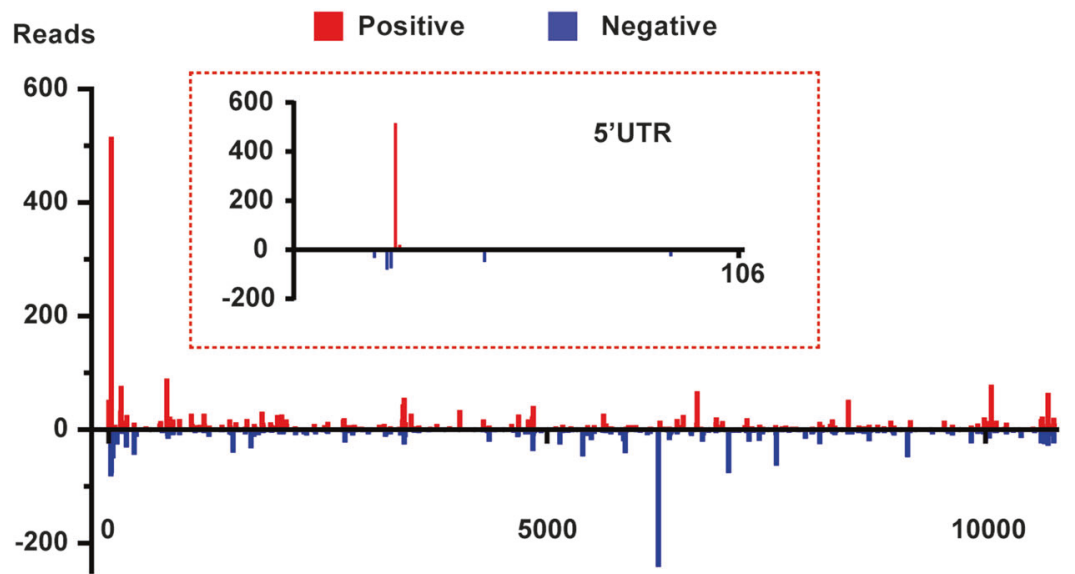

ZIKV genome (nt) b

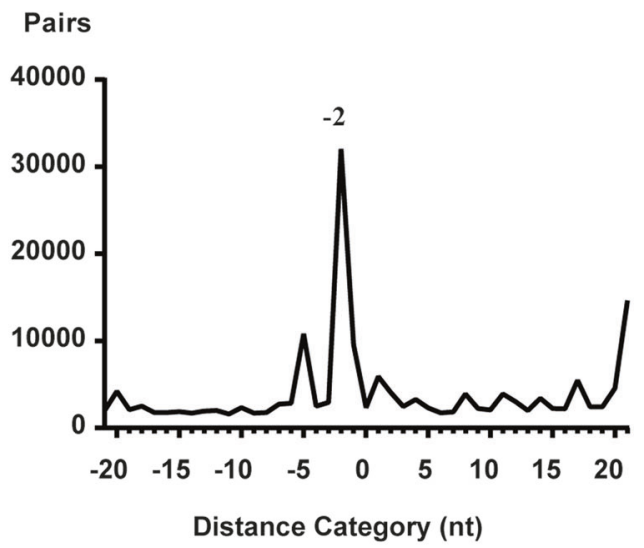

e

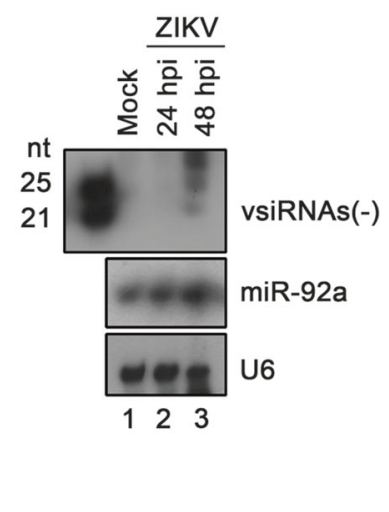

d

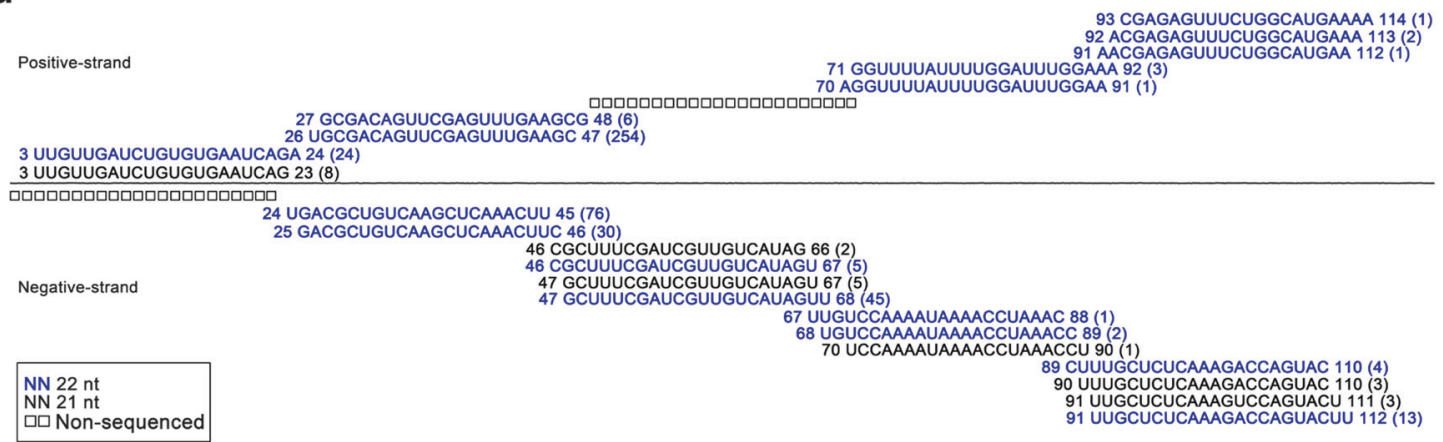

Fig. 1 Production of ZIKV-derived siRNAs in hNPCs. hNPCs were infected with ZIKV (strain GZ01) at an MOI of 2 and total RNAs were subjected to deep sequencing. a Size distribution and abundance (counts per million of total reads, CPM) of total vsRNAs sequenced from the ZIKVinfected hNPCs. Red, positive-stranded vsRNAs; blue, negative-stranded vsRNAs. b Total pairs of complementary 22-nt vsRNAs derived from ZIKV in each distance category between the $5^{\prime}$ - and 3'-ends of a complementary vsRNA pair. "-2" is shown for pairs with a 2-nt overhang at the $3^{\prime}$-end of each strand, which is defined as the canonical viral siRNAs (vsiRNAs). c Distribution of vsRNA reads (21-23 nt in size) in the positive- and negative-stranded ZIKV genome. The relative abundances of positive- and negative-stranded vsRNAs are indicated. The experiments were repeated two times, and representative results are shown. $\mathbf{d}$ Read sequences along the indicated segments of the ZIKV genome. Read counts (in brackets), read length and genomic position are indicated. e Northern blot assay of vsRNAs in ZIKV-infected hNPCs. miR-92a and U6 were used as internal references

HEK293T cells; enoxacin treatment dramatically enhanced both shRNA- and siRNA-mediated silencing of EGFP (Supplementary information, Fig. S6a), but did not affect the production of siRNAs from shRNAs (Supplementary information, Fig. S6b), confirming that enoxacin functions at steps post siRNA production by Dicer. ${ }^{47,49}$ Initially, we assayed the cytotoxicity of enoxacin in hNPCs by standard cell viability assays, and the $50 \%$ cytotoxic concentration $\left(\mathrm{CC}_{50}\right)$ was calculated to $175.8 \mu \mathrm{M}$ (Supplementary 
a

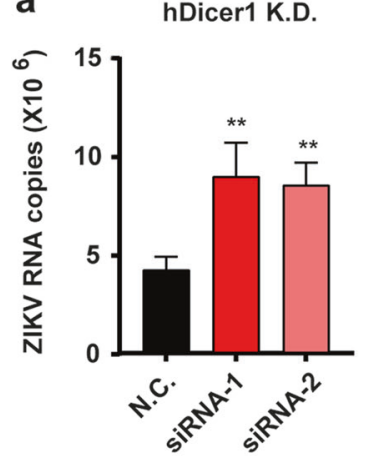

C

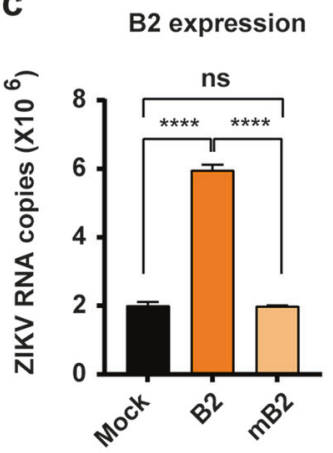

hAgo2 K.D.

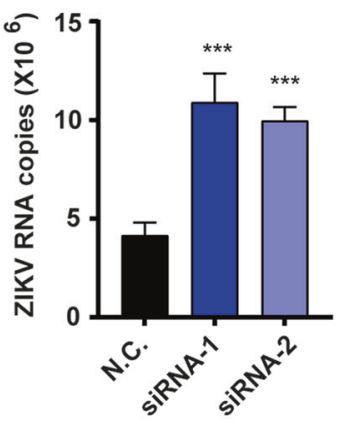

d

siRNA targeting ZIKV

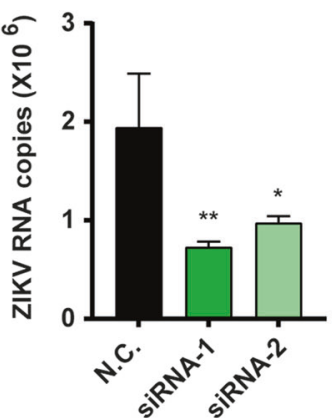

b
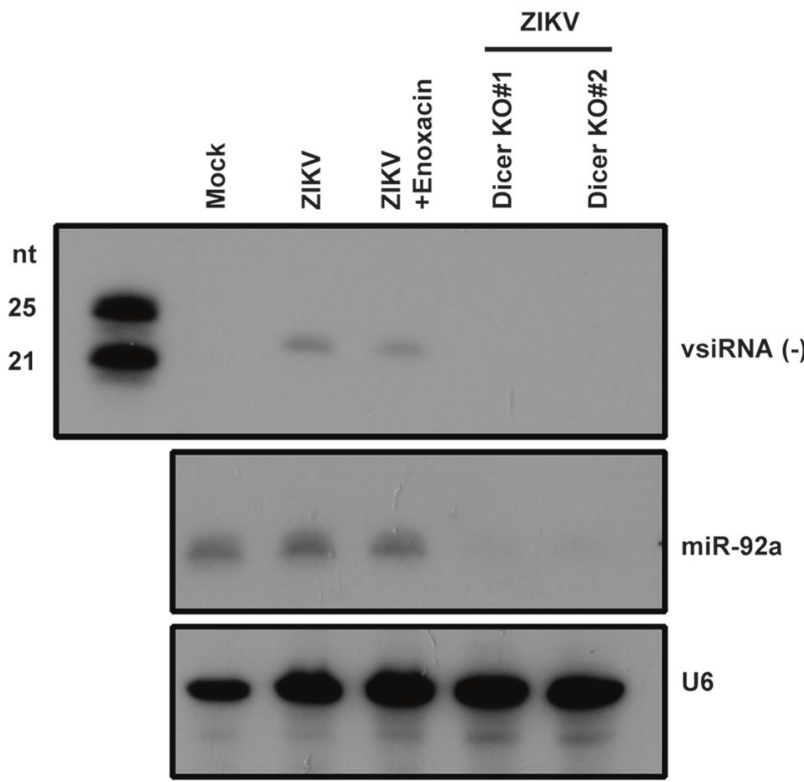

Fig. 2 RNAi plays antiviral roles in ZIKV-infected hNPCs. a hNPCs in which Dicer1 or Ago2 were knocked down (K.D.) via the corresponding siRNAs (siRNA-1 and siRNA-2, $25 \mathrm{nM}$ ) or treated with a specific control siRNA (N.C., $25 \mathrm{nM}$ ) were infected with ZIKV at an MOI of 0.02 . Fortyeight hours later, the amounts of ZIKV genomic RNA in each culture supernatant was determined by RT-qPCR. b hNPC or Dicer KO cell lines were infected with ZIKV at an MOI of 2. Northern blot assay of vsRNAs in cells at 48 hpi. miR-92a and U6 were used as internal references. c hNPCs that stably express Nov B2 or mB2 were infected with ZIKV at an MOI of 0.02 . The amounts of ZIKV genomic RNA in the culture supernatants were determined by RT-qPCR at $48 \mathrm{hpi}$. $\mathbf{d}$ hNPCs treated with N.C. siRNA or ZIKV-specific siRNAs (10 $\mu \mathrm{M})$ were infected with ZIKV at an $\mathrm{MOI}$ of 0.02 and analyzed using a ZIKV-specific RT-qPCR assay. All data are expressed as the means \pm SD of three independent experiments. Statistical significance was determined by a Student's $t$-test using GraphPad Prism. ${ }^{*} P<0.05,{ }^{* *} P<0.01,{ }^{* * *} P<0.001,{ }^{* * * *} P<$ 0.0001

information, Fig. S7). Strikingly, $100 \mu \mathrm{M}$ of enoxacin efficiently prevented ZIKV infection in hNPCs (Fig. 3a), and both Asian and African ZIKV strains were sensitive to the antiviral effect of enoxacin (Fig. 3b). The $50 \%$ inhibition concentration $\left(\mathrm{IC}_{50}\right)$ of enoxacin was calculated as $51.99 \mu \mathrm{M}$ in hNPCs (Fig. 3c). As expected, enoxacin failed to prevent ZIKV infection in both Dicer KO hNPCs (Supplementary information, Fig. S8a) due to the lack of vsiRNAs (Fig. 2b). In addition, enoxacin showed no antiviral effects in the Dicer-deficient 293T (293T-NoDice) cells ${ }^{36}$ and human RD cells (Supplementary information, Fig. S8b). More importantly, enoxacin exerted similar anti-ZIKV effects in the hNPC line 15167 (Supplementary information, Fig. S9), which was originally isolated from a human fetal brain. ${ }^{50}$ In addition, exogenous vsiRNA mimics showed anti-ZIKV activity in 293 T cells, and enoxacin significantly enhanced the antiviral effect (Supplementary information, Fig. S10). Collectively, these results demonstrate that enoxacin can be used to inhibit ZIKV infection in an RNAi-dependent manner.

Brain-region-specific organoids are capable of recapitulating much of the composition, diversity and organization of cell types found in the developing human fetal brain, and have been extensively employed to model ZIKV-associated microcephaly and evaluate antiviral drugs. ${ }^{11,51}$ We finally assayed the antiviral effects of enoxacin in two sets of brain organoids at different developmental stages. The hESC-derived cortical organoids of D20 contain structures representing ventricular zone (VZ) and subventricular zones (SVZ) where hNPCs predominantly reside, while those of D38 bear a lumen and more mature neuronal layers. For the D20 brain organoids, ZIKV predominantly infected SOX2 ${ }^{+}$hNPCs in the VZ and SVZ (Fig. 4a and Supplementary information, Fig. S11), causing thinning of the VZ layer and smaller organoids (Fig. 4a, b) that imitates the events in fetal microcephaly. Remarkably, enoxacin treatment of the D20 brain organoids effectively attenuated ZIKV replication (Fig. 4a), which in turn restored normal cell proliferation (Supplementary information, Fig. S11b) with no detrimental effects on the thickness of VZ layer or the size of organoids (Fig. 4a, b). For the D38 brain organoids, ZIKV infection caused disorganization of the VZ and SVZ, loss of lumen and massive cell death (Supplementary information, Fig. S12), while enoxacin treatment rescued hNPC proliferation and maintained the well-organized lumen and cell layers (Supplementary information, Fig. S12). Collectively, these results demonstrate that enoxacin could completely prevent ZIKV infection in hNPCs, thus circumvent ZIKV-induced damage even in the more complex brain organoids that contain multiple cell types other than hNPCs.

\section{DISCUSSIONS}

RNAi has long been established as a major antiviral defense mechanism in fungi, plants and invertebrates. However, the importance of RNAi as a mammalian antiviral defense mechanism has been questioned due to the absence of vsiRNAs in mammalian cells infected with a wild-type human virus. ${ }^{31}$ Here, we show that ZIKV infection readily triggered the abundant production of vsiRNAs in hNPCs, with a similar potency to in mosquito Aag2 cells. Previously, we and others have demonstrated that human EV71 and IAV encoded specific VSRs to antagonize antiviral RNAi pathway, thus vsiRNAs only became detectable in mammalian somatic cells infected 
a
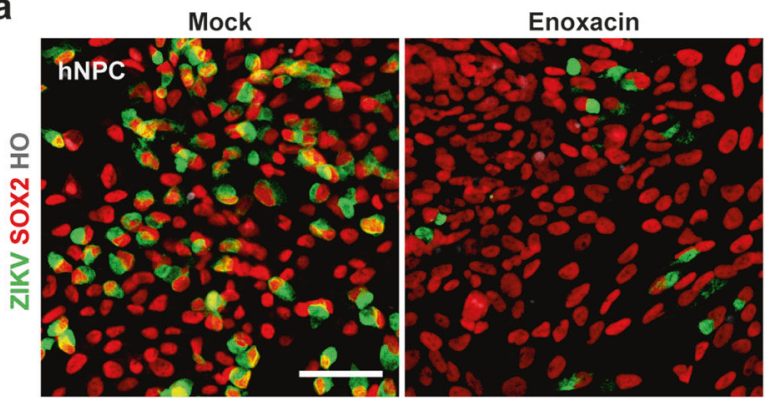

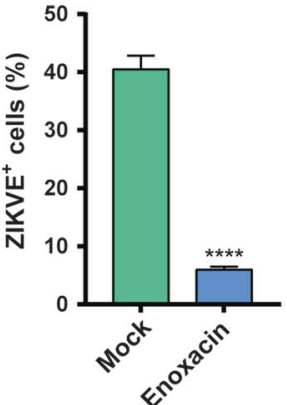

C

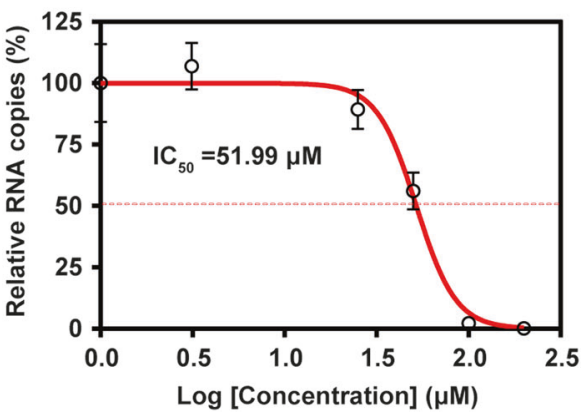

b

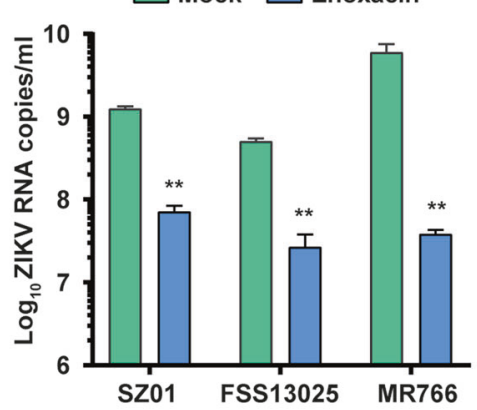

b

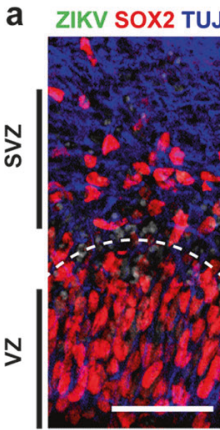

Mock

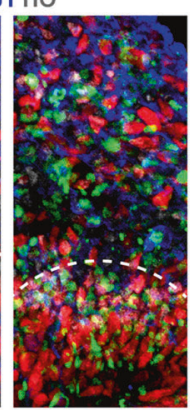

ZIKV

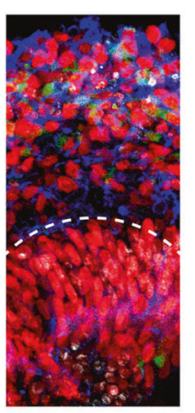

ZIKV +Enoxacin
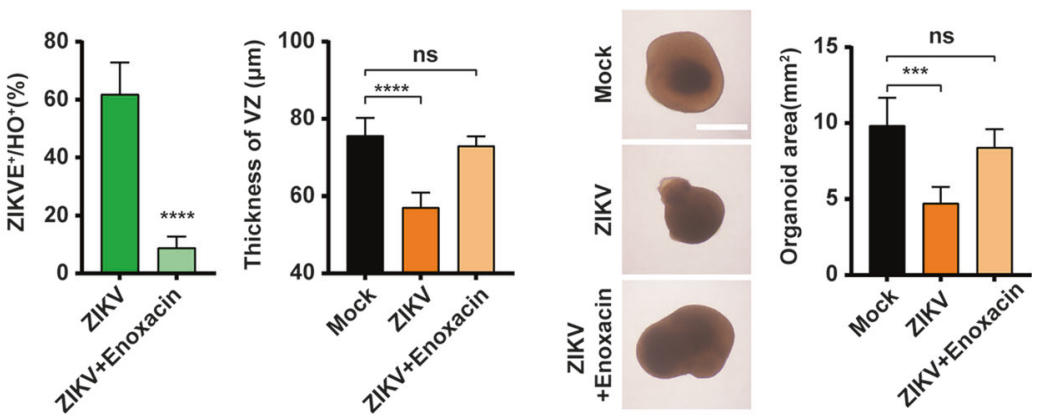

Fig. 4 Enoxacin rescues ZIKV-caused microcephaly in brain organoids. Following ZIKV infection, D20 brain organoids treated with enoxacin were subjected to immunostaining and virological analysis. a Enoxacin treatment prevented ZIKV infection and the thinning of the VZ layer in the D20 organoids as indicated by the white dotted lines. ZIKV E (green), SOX2 (red), TUJ1 (blue). ZIKVE ${ }^{+}$cells and the thickness of the SOX2 ${ }^{+}$ VZ layer were quantified. Scale bar, $50 \mu \mathrm{m}$. b Morphologic demonstration of the organoids in each group. Surface areas of organoids were quantified. All data are means \pm SD ( $n=5$ organoids) of three independent experiments, Student's $t$-test and one-way ANOVA, $* * * P<0.001$, $* * * P<0.0001$. Scale bar, $200 \mu \mathrm{m}$

with VSR-defective mutant viruses. ${ }^{29,30,52}$ Actually, some flavivirus proteins or RNA elements, e.g., the capsid and specific non-coding RNAs, have been characterized in vitro to have potential VSR activity. ${ }^{53,54} \mathrm{~A}$ recent integrated proteomic analysis has also predicated the potential interaction between ZIKV capsid protein and Dicer in hNPCs. ${ }^{55}$ Thus, ZIKV probably has also evolved to encode some specific VSR to antagonize the RNAi pathway in hNPCs or other types of human cells, and the underlying mechanism is under investigation.

Moreover, our results showed that knockdown of the RNAi machinery components or suppression of RNAi by the known VSR efficiently promoted ZIKV replication in hNPCs (Fig. 2). These results further intensified the concept that hNPCs use RNAi as an effective antiviral defense against an authentic human pathogenic virus, ZIKV. Meanwhile, the absence of vsiRNAs in the more differentiated human neurons and somatic cells suggested a decay in RNAi-mediated antiviral immunity during neural development. It should be noted that hNPCs not only play critical roles during fetal brain development, but also persist in adult brain throughout human life span. ${ }^{56}$ Thus, the evolutionarily ancient antiviral mechanism, RNAi, remains an active antiviral immune defense in human, especially, in specific cell populations like hNPCs. Interestingly, a previous study by Maillard et al. reported that murine ESCs (mESCs) infected by encephalomyocarditis virus 
(EMCV) can produce vsiRNAs, indicating a potential function of RNAi pathway in mESCs. ${ }^{27}$ However, EMCV is a cardiovirus that causes encephalomyocarditis in pigs, but not a natural pathogen of $m E S C s$. These findings obtained by using EMCV-infected mESCs thus may not simply apply to hNPCs and other human cells.

Interestingly, Rice and colleagues showed that hESCs and some tissue stem cells are deficient in inducible IFN responses, but intrinsically express cell type-specific subsets of ISGs, ${ }^{57}$ consistent with the idea that the canonical IFN-inducible ISG expressions are unfavorable for both RNAi-mediated antiviral immunity and the maintenance of stem cell niche. Some early investigations have suggested that the IFN-based antiviral immunity in mammals may inherently conflict the antiviral RNAi system. ${ }^{58,59}$ Therefore, a reasonable explanation about why antiviral RNAi become physiologically important in hNPCs is probably due to the less potency of hNPCs in IFN-based immune responses compared to the more differentiated neurons and other somatic cells. Moreover, previous studies have shown that RNAi can play potent antiviral roles even in mammalian somatic cells defective in type I IFN signaling, indicating that antiviral RNAi can be functionally independent of type I IFN response, ${ }^{29,60}$ which is consistent with the notion that antiviral RNAi is physiologically important in hNPCs that have reduced IFN response.

The identification of antiviral RNAi response in hNPCs leads to the exciting concept that ZIKV replication and pathogenesis in hNPCs may be therapeutically intervened by enhancing RNAi. Enoxacin is an FDA-approved fluoroquinolone antibiotic, which has never been linked to antiviral activity. Several independent groups have identified its RNAi-enhancing capability, and enoxacin treatment increases the efficiency of exogenous siRNAand shRNA-mediated gene silencing. ${ }^{47,48}$ In our study, we showed that enoxacin is a potent antiviral agents in hNPCs and Aag2 cells that are capable of producing vsiRNAs (Fig. 3). By contrast, in the more differentiated neurons or No-Dicer cells that are incapable of processing ZIKV dsRNA into vsiRNA, enoxacin treatment showed no antiviral activity. Furthermore, in the more complex cortical organoids that contain multiple cell types other than hNPCs, enoxacin treatment showed significant antiviral effects and fully rescued ZIKV-induced microcephaly phenotype. These promising results in hNPCs and brain organoids warrant further investigation into the therapeutic potential of enoxacin in clinical trials. Most importantly, this proof-of-concept novel antiviral strategy may be adapted to treat other types of congenital viral infection, should additional types of cells that employ RNAi as an antiviral defense be identified and additional compounds with better RNAienhancing activity be discovered.

\section{MATERIALS AND METHODS}

Cells

Human RD, A549, HEK293T cells and BHK-21 cells were obtained commercially from the ATCC and cultured in Dulbecco's modified eagle medium (DMEM, Thermo Fisher Scientific) containing 10\% fetal bovine serum (FBS, Biowest), and supplemented with $100 \mathrm{U} /$ $\mathrm{mL}$ penicillin and $100 \mathrm{mg} / \mathrm{mL}$ streptomycin at $37^{\circ} \mathrm{C}$ in a humidified atmosphere with $5 \% \mathrm{CO}_{2}$. The NoDice $293 \mathrm{~T}$ cell line ${ }^{36}$ was generously provided by Dr. Bryan R. Cullen (Duke University Medical Center, Durham, NC, USA). The Aedes aegypti cell line Aag2 (ATCC CCL-125) was cultured in Schneider's Drosophila medium (Thermo Fisher Scientific) containing $10 \%$ FBS at $30^{\circ} \mathrm{C}$. The Aedes albopictus cell line C6/36 (ATCC CRL-1660) was cultured in RPMI 1640 (Thermo Fisher Scientific) medium containing 10\% FBS at $30^{\circ} \mathrm{C}$.

The human H9 (WA09) ESC line was from WiCell Research Institute and maintained on Matrigel (Corning) using E8 (Thermo Fisher Scientific) and passaged using EDTA $(100 \mu \mathrm{M})$. Neural differentiation of the $\mathrm{H} 9$ cell line was performed using embryoid bodies (EBs) as described previously with minor modifications. ${ }^{61}$
Briefly, hESC colonies were detached by incubating with dispase $(1 \mathrm{mg} / \mathrm{mL}$ ) and suspended as cell aggregates in the neural induction medium (NIM) consisting of DMEM/F12 (Thermo Fisher Scientific), N2 supplement (Thermo Fisher Scientific), MEM nonessential amino acids (Thermo Fisher Scientific) and heparin (Sigma, $2 \mu \mathrm{g} / \mathrm{mL}$ ). EBs were attached to $100 \mathrm{~mm}$ Petri dishes in NIM on day 7. EBs could form typical columnar neuroepithelia, which were organized into neural rosettes between day 10 and day 12 . On day 15 , the neural rosettes were detached by pipetting and suspended in NIM as neuroepithelial spheres. By day 25, neuroepithelial spheres were differentiated into dorsal telencephalic progenitor cells and dissociated with Accutase (Thermo Fisher Scientific, $1 \mathrm{mg} / \mathrm{mL}$ ). hNPCs were plated onto Matrigelcoated coverslips in NIM and used for ZIKV infection. For neuronal differentiation, neural progenitors' clusters were dissociated with Accutase and plated onto ornithine/laminin-coated coverslips (Stem Cell Technologies) on day 25 in neurobasal medium (Thermo Fisher Scientific) supplemented with brain-derived neurotrophic factor (BDNF, Peprotech, $20 \mathrm{ng} / \mathrm{mL}$ ), glial-derived neurotrophic factor (GDNF, Peprotech, $10 \mathrm{ng} / \mathrm{mL}$ ), insulin-like growth factor 1 (IGF1, Peprotech, $10 \mathrm{ng} / \mathrm{mL}$ ), and CAMP (Stem Cell Technologies, $1 \mu \mathrm{M})$. On day 35, dorsal telencephalic neurons were characterized with specific markers and used for ZIKV infection.

hNPC line 15167 derived from fetal brains (Lonza) was kindly provided by S. Bao (Cleveland Clinic) ${ }^{50}$ and cultured as neurospheres in neurobasal complete medium (Gibco) supplemented with B-27 supplement without Vitamin A (Thermo Fisher Scientific), L-glutamine (MACGENE, $2 \mathrm{mM}$ ), sodium pyruvate (MACGENE, $1 \mathrm{mM}$ ), basic fibroblast growth factor (bFGF, R\&D Systems, $10 \mathrm{ng} / \mathrm{mL}$ ), and epidermal growth factor $(E G F, R \& D$ Systems, $10 \mathrm{ng} / \mathrm{mL}$ ).

\section{Viruses}

Three Asian lineage ZIKV strains were used in this study: ZIKV strain GZ01 (GenBank number: KU820898) isolated from a patient returning from Venezuela in 2016, ${ }^{62}$ ZIKV strain SZ01 (GenBank number: KU866423) isolated from a patient returning from Samoa in 2016, ${ }^{63}$ and strain FSS13025 (GenBank number: KU955593) isolated from a patient in Cambodia in 2016. ${ }^{64}$ ZIKV strain MR766 (GenBank number: AY632535), the representative strain of African lineage, was kindly provided by Prof. Aihua Zheng (Institute of Zoology, CAS). ${ }^{65}$ All ZIKV stocks were prepared in C6/36 cells and titrated by plaque assay on BHK-21 cells as described below. Studies with infectious ZIKV were conducted under biosafety level 2 (BSL-2) conditions at the Beijing Institute of Microbiology and Epidemiology with approval from Institutional Biosafety Committee.

\section{Generation of DICER KO hNPC clones}

Standard CRISPR/Cas9 method was used to generate the Dicer KO hNPCs. Briefly, three gRNAs were designed and cloned (Supplementary information, Table S2), respectively, and co-transfected with the pCAG-CAS9-2A-EGFP plasmid (kindly provided by Prof. Wei Li from Institute of Zoology, CAS) into hNPCs using Lipofectamine $^{\mathrm{TM}}$ Stem Transfection Reagent (Thermo Fisher Scientific). EGFP-positive cells were sorted by FACS and plated into 96-well plates with one cell per well. Then, two posive clones were characterized by Sanger sequencing (Supplementary information, Table S2), western blotting and immunostaining assays.

Lentivirus production and transduction of hNPCs

The NoV B2 (B2) and mutant Nov B2 (mB2) genes ${ }^{45}$ were subcloned into the pLVX-EF1a-IRES-Neo vector (Clontech). To produce the lentivirus stock, the resulting constructs were cotransfected into HEK293T cells together with the packaging vectors, psPAX2 and pMD2.G. Forty-eight hours later, the cell culture medium was collected and viruses were further 
concentrated by ultrafiltration (Millipore, U-15). For transduction, concentrated viruses were added into the hNPC medium (MOI = 10) and incubated with polybrene (Sigma, $8 \mu \mathrm{g} / \mathrm{mL}$ ) for $24 \mathrm{~h}$. After transduction under selection with G418 (Sigma, $400 \mu \mathrm{g} / \mathrm{mL}$ ) for 2 weeks, the hNPCs that stably expressed B2 and mNB2 were ready for infection with ZIKV.

Human brain-specific organoid model

Human brain-specific organoids were generated from H9 ESCs as previously described. ${ }^{66}$ In brief, hESCs were maintained on Matrigel-coated plates. Then hESC clones were dissociated using dispase (Invitrogen), and suspended into ultra-low attachment $100 \mathrm{~mm}$ plastic plates (Corning) to form pre-organoids. Cells were then maintained for 7 days in medium containing DMEM/F12, N2 supplement, non-essential amino acids, GlutaMAX (Thermo Fisher Scientific), Heparin, IWR-1-endo (Merck) and SB-431542 (Merck). Notably, the medium was supplemented with ROCK inhibitor Y-27632 (Stem Cell Technologies, $10 \mu \mathrm{M}$ ) for the first $24 \mathrm{~h}$. On day 8 of the protocol, floating spheroids were transferred to neural medium consisting of neurobasal medium, B-27 supplement (Thermo Fisher Scientific) and GlutaMAX supplemented with bFGF (Peprotech, $10 \mathrm{ng} / \mathrm{mL}$ ) and EGF (Peprotech, $20 \mathrm{ng} / \mathrm{mL}$ ), with medium change every other day (Day 8 to Day 22).

In our system, the D20 brain-specific organoids differentiated into distinct VZ and SVZ. Starting on day 23, organoids were cultured in neurobasal medium with BDNF (Peprotech, $10 \mathrm{ng} / \mathrm{mL}$ ), GDNF (Peprotech, $10 \mathrm{ng} / \mathrm{mL}$ ), IGF1 (Peprotech, $10 \mathrm{ng} / \mathrm{mL}$ ) and Neurotrophin-3 (NT3, Peprotech, $20 \mathrm{ng} / \mathrm{mL}$ ). The NPC area was clearly distinguished from the neuronal area in the D38 brainspecific organoids.

Quantification of the average diameter of the organoids: $a$, long semi-axis; $b$, short semi-axis. Average diameter $c=2 \times \sqrt{a \times b}$. Layer thickness and area were measured by scale, and percentage of cells was counted by ImageJ (National Institutes of Health).

RNA isolation and determination

Viral or cellular RNAs were extracted using the Purelink RNA mini kit (Thermo Fisher Scientific) according to the manufacturer's instructions. ZIKV genomic RNA was quantified by one step PrimeScript $^{\text {TM }}$ RT-qPCR Kit (Takara). IFN pathway-related gene transcripts were quantified using a one-step SYBR green RT-PCR Kit (Takara) using the probe and primers listed in Supplementary information, Table S3.

Deep sequencing and data analyses

Total RNAs from the ZIKV-infected cells were extracted by Trizol. Libraries of small RNAs extracted from cell cultures were constructed using TruSeq Small RNA Library Preparation Kits (Illumina) according to the manufacturer's instruction. The libraries were sequenced using an Illumina HiSeq 2000 at RiboBio. The sequencing reads were mapped to the human genome (release version hg19) using Bowtie 2.2.5 and the cellular miRNA expression level was determined on the basis of the number of reads that were identical to the annotated miRNA sequences in miRBase (version 18), only allowing -2 or +2 nt to be templated by the corresponding genomic sequence at the $3^{\prime}$ ends. The small RNA reads that could not be mapped to the human genome were then mapped to ZIKV genome with $100 \%$ identity. The subsequent analysis of the length and location of these vsRNAs were calculated using in-house Perl scripts. ${ }^{29}$ Pairs of complementary 22-nt vsRNAs in each library in different distance categories were computed using principles as previously described. ${ }^{28}$

Northern blot assay

For northern blot analysis of low-molecular-weight (LMW) RNA, 20 $\mu \mathrm{g}$ of small RNA-enriched total RNAs were resolved by $7 \mathrm{M}$ urea$15 \%$ PAGE, transferred to a Hybond-A nylon membrane (GE
Healthcare) and chemically cross-linked using 1-ethyl-3-(3dimethylaminopropyl) carbodiimide (EDC) as previously described. $^{29}$ The probes for detection of ZIKV-derived vsRNAs were complementary to nucleotides $1-50$ of the ZIKV antigenome. The RNA probes were simultaneously produced and labeled with DIG-UTP (Roche) by in vitro transcription. For detection of small RNAs, DIG-labeled oligo RNA probes targeting the EGFP siRNAs, and miR-92a and U6 were synthesized by Takara (Supplementary information, Table S4).

\section{Cytokine analysis}

The cell culture supernatants collected from ZIKV-infected cells at 48 hpi were tested by Human Cytokine Magnetic 25-Plex Panel (Thermo Fisher Scientific) according to the manufacturer's instructions. The data were collected on Luminex $200^{\mathrm{TM}}$ and analyzed by Luminex xPONENT (Thermo Fisher Scientific).

Enoxacin treatment

Enoxacin was dissolved in DMSO. For cell proliferation assay, hNPCs were incubated with enoxacin dilutions from $1 \mathrm{mM}$ to $3.90625 \mu \mathrm{M}$ in 96 -well plates. Then cell viability were determined using the Cell Titer-Glo Luminescent Cell Viability Assay kit (Promega). The $\mathrm{CC}_{50}$ was calculated by GraphPad Prism 7.01. To assay the antiviral effects of enoxacin, various cells were treated with enoxacin $(100 \mu \mathrm{M})$ for $24 \mathrm{~h}$ and then infected with ZIKV at an $\mathrm{MOI}$ of 2 . After $1 \mathrm{~h}$, the medium was replaced with fresh medium containing $100 \mu \mathrm{M}$ enoxacin. At $48 \mathrm{hpi}$, cell culture supernatants were collected to detect viral RNA copies and cells were fixed for immunofluorescence assay. To determine the $I_{50}$ of enoxacin, hNPCs were treated with enoxacin at 2-fold diluent concentrations from 200 to $3.125 \mu \mathrm{M}$. The $\mathrm{IC}_{50}$ was calculated by GraphPad Prism 7.01 .

Plaque forming assays

Virus titers were determined by plaque forming assay in BHK-21 cells. Briefly, BHK-21 cells were seeded in a 12-well plate for $24 \mathrm{~h}$, and cells were infected with $400 \mu \mathrm{L}$ of 10 -fold viral dilutions. Viral supernatants were replaced with DMEM containing 1\% low melting point agarose (Promega) and 1\% FBS. Viral plaques became detectable at 4-5 dpi. Cells were then fixed with $4 \%$ formaldehyde, followed by staining with $1 \%$ crystal violet solution. Finally, all visible plaques were counted, and the final titers were calculated accordingly.

\section{ZIKV NS1 ELISA}

ZIKV NS1 in cell culture supernatants was measured using a commercial ELISA Kit (BioFront). Briefly, samples were fourfold diluted using the dilution buffer, and the optical density (OD) was measured at $450 \mathrm{~nm}$ with an ELISA reader (Beckman). The concentration of ZIKV NS1 was calculated according to the manufacturer's instruction.

Immunofluorescence assay

Cells were fixed with $4 \%(\mathrm{w} / \mathrm{v})$ paraformaldehyde in PBS at room temperature (RT) for $15 \mathrm{~min}$. Organoids were incubated in a $30 \%$ $(\mathrm{w} / \mathrm{v})$ sucrose solution overnight at $4{ }^{\circ} \mathrm{C}$ and placed in tissue base molds and embedded within an Optical coherence tomography (O.C.T) compound (Tissue-Tek, Hatfield, PA, USA) at $-20^{\circ} \mathrm{C}$. Organoid blocks were then used for cryosectioning to obtain 20 $\mu \mathrm{m}$ slices using a freezing microtome (Leica) or stored at $-80^{\circ} \mathrm{C}$. Cryosections were washed with washing buffer $(1 \times \mathrm{PBS}, 0.3 \%$ Triton X-100) for 3 times ( 5 min for each time) at RT, and then fixed with $4 \%$ paraformaldehyde in PBS for $15 \mathrm{~min}$.

Cells and cryosections of organoids were blocked in PBS buffer containing 10\% donkey serum and $0.3 \%$ Triton X-100 (Sigma) for $1 \mathrm{~h}$ at RT, followed by incubation with the primary antibodies at $4{ }^{\circ} \mathrm{C}$ overnight with $5 \%$ donkey serum and $0.15 \%$ Triton X-100. Cryosections were incubated with secondary antibodies diluted in 
$5 \%$ donkey serum and $0.15 \%$ Triton $\mathrm{X}-100$ for $1 \mathrm{~h}$. Nuclei were counterstained with Hoechst 33342 DNA dye (Life Technologies, 1: $1,000)$ at RT for 10 min and mounted on glass slides. Images were taken using a Carl Zeiss LSM710 confocal microscope and processed using ZEN 2012 software. The following primary antibodies were used for immunofluorescence: ZIKV E protein (BioFront, BF-1176-66, mouse, 1:1,000), human SOX2 (R\&D, AF2018, goat, 1:1,000), human Tuj1 (Sigma, T2200, rabbit, 1:1,000), human NESTIN (Santa Cruz Biotechnology, sc-20978, rabbit, 1:1,000), cleaved Caspase-3 (Cell Signaling Technology, CST-9661, rabbit, 1:1,000). EdU ${ }^{+}$cells was detected by Click-iT EdU Imaging Kits (Invitrogen) according to the manufacturer's protocol.

Statistical analysis

All data were analyzed using the GraphPad Prism 7.01 software. For the statistical analysis of other results, statistical evaluation was performed by Student's unpaired $t$-test or one-way ANOVA with Tukey's multiple comparisons test. Data are presented as means \pm SD or as described in the corresponding legends.

Data availability

The RNA-seq data has been deposited to the NCBI GEO database under the accession number: GSE125520.

\section{ACKNOWLEDGEMENTS}

We thank Drs. B.R. Cullen (Duke University Medical Center), S. Bao (Cleveland Clinic), W. Li (CAS), and A. Zheng (CAS) for critical reagents, and A.D. Davidson (University of Bristol) for proofreading the manuscript. This work was supported by the National Key Research and Development Project of China (2016YFD0500304), the Research Program of CAS (XDA16020604), the Strategic Priority Research Program of CAS (XDPB0301), the National Science and Technology Major Project of China (2018ZX09711003, 2018ZX10101004 and 2017ZX10304402), the National Natural Science Foundation of China (81873964, 31770190, 31730108, 81661148054, $81661130162,81621005,81522025$ and 31522004). C.-F.Q. and X.Z. were supported by the Newton Advanced Fellowship from the UK Academy of Medical Sciences. J.C. was supported by funding from the CAS Pioneer Hundred Talents Program.

\section{AUTHOR CONTRIBUTIONS}

C.-F.Q., X.Z. and B.H. designed the study and wrote the paper. Y.-P.X., Y.Q., B.Z. and G.C. performed the majority of the experiments and analyzed the data; Q.C. and M.W., F.M., J.X., J.W., R.-R.Z., M.-L.C., N.-N. Z., B.L., W.-L.Z., M.-H.W., Q.Y., D.Z., J.-H.M., X.-F.L., J.C. and Z.X. contributed specific experiments and data analysis. All authors read and approved the contents of the manuscript.

\section{ADDITIONAL INFORMATION}

Supplementary information accompanies this paper at https://doi.org/10.1038/ s41422-019-0152-9.

Competing interests: The authors declare no competing interests.

\section{REFERENCES}

1. Dick, G. W., Kitchen, S. F. \& Haddow, A. J. Zika virus. I. Isolations and serological specificity. Trans. R. Soc. Trop. Med. Hyg. 46, 509-520 (1952).

2. Weaver, S. C. et al. Zika virus: history, emergence, biology, and prospects for control. Antivir. Res. 130, 69-80 (2016).

3. Baud, D., Gubler, D. J., Schaub, B., Lanteri, M. C. \& Musso, D. An update on Zika virus infection. Lancet 390, 2099-2109 (2017).

4. Pierson, T. C. \& Diamond, M. S. The emergence of Zika virus and its new clinical syndromes. Nature 560, 573-581 (2018).

5. Musso, D. \& Gubler, D. J. Zika virus. Clin. Microbiol. Rev. 29, 487-524 (2016).

6. de Araujo, T. V. B. et al. Association between Zika virus infection and microcephaly in Brazil, January to May, 2016: preliminary report of a case-control study. Lancet Infect. Dis. 16, 1356-1363 (2016).

7. Orioli, I. M. et al. Prevalence and clinical profile of microcephaly in South America pre-Zika, 2005-2014: prevalence and case-control study. Br. Med. J 359, j5018 (2017).
8. Tang, H. et al. Zika virus infects human cortical neural progenitors and attenuates their growth. Cell Stem Cell 18, 587-590 (2016).

9. Dang, J. et al. Zika virus depletes neural progenitors in human cerebral organoids through activation of the innate immune receptor TLR3. Cell Stem Cell 19, 258-265 (2016).

10. Garcez, P. P. et al. Zika virus impairs growth in human neurospheres and brain organoids. Science 352, 816-818 (2016).

11. Qian, X. et al. Brain-region-specific organoids using mini-bioreactors for modeling ZIKV exposure. Cell 165, 1238-1254 (2016).

12. Wu, K. Y. et al. Vertical transmission of Zika virus targeting the radial glial cells affects cortex development of offspring mice. Cell Res. 26, 645-654 (2016).

13. Cugola, F. R. et al. The Brazilian Zika virus strain causes birth defects in experimental models. Nature 534, 267-271 (2016).

14. Li, C. et al. Zika virus disrupts neural progenitor development and leads to microcephaly in mice. Cell Stem Cell 19, 672 (2016).

15. Muffat, J. et al. Human induced pluripotent stem cell-derived glial cells and neural progenitors display divergent responses to Zika and dengue infections. Proc. Natl Acad. Sci. USA 115, 7117-7122 (2018).

16. Wells, M. F. et al. Genetic ablation of AXL does not protect human neural progenitor cells and cerebral organoids from Zika virus infection. Cell Stem Cell 19, 703-708 (2016).

17. Wen, Z., Song, H. \& Ming, G. L. How does Zika virus cause microcephaly? Genes Dev. 31, 849-861 (2017).

18. Caires-Junior, L. C. et al. Discordant congenital Zika syndrome twins show differential in vitro viral susceptibility of neural progenitor cells. Nat. Commun. 9, 475 (2018).

19. $\mathrm{Xu}, \mathrm{M}$. et al. Identification of small-molecule inhibitors of Zika virus infection and induced neural cell death via a drug repurposing screen. Nat. Med. 22, 1101-1107 (2016).

20. $\mathrm{Li}$, Z. et al. Existing drugs as broad-spectrum and potent inhibitors for Zika virus by targeting NS2B-NS3 interaction. Cell Res. 27, 1046-1064 (2017).

21. Schneider, W. M., Chevillotte, M. D. \& Rice, C. M. Interferon-stimulated genes: a complex web of host defenses. Annu. Rev. Immunol. 32, 513-545 (2014).

22. Guo, Y. L. Utilization of different anti-viral mechanisms by mammalian embryonic stem cells and differentiated cells. Immunol. Cell Biol. 95, 17-23 (2017).

23. Van der Hoek, K. H. et al. Viperin is an important host restriction factor in control of Zika virus infection. Sci. Rep. 7, 4475 (2017).

24. Zhang, F. et al. Molecular signatures associated with ZIKV exposure in human cortical neural progenitors. Nucleic Acids Res. 44, 8610-8620 (2016).

25. Guo, Z., Li, Y. \& Ding, S. W. Small RNA-based antimicrobial immunity. Nat. Rev. Immunol. 19, 31-44 (2018).

26. Samuel, G. H., Adelman, Z. N. \& Myles, K. M. Antiviral immunity and virusmediated antagonism in disease vector mosquitoes. Trends Microbiol. 26, 447-461 (2018).

27. Maillard, P. V. et al. Antiviral RNA interference in mammalian cells. Science $\mathbf{3 4 2}$, 235-238 (2013)

28. Li, Y., Lu, J., Han, Y., Fan, X. \& Ding, S. W. RNA interference functions as an antiviral immunity mechanism in mammals. Science 342, 231-234 (2013).

29. Qiu, Y. et al. Human virus-derived small RNAs can confer antiviral immunity in mammals. Immunity 46, 992-1004 (2017).

30. Soares de Souza, A. et al. Fetal infection by Zika virus in the third trimester: report of 2 cases. Clin. Infect. Dis. 63, 1622-1625 (2016).

31. Cullen, B. R., Cherry, S. \& tenOever, B. R. Is RNA interference a physiologically relevant innate antiviral immune response in mammals? Cell Host Microbe 14, 374-378 (2013).

32. Berkhout, B. RNAi-mediated antiviral immunity in mammals. Curr. Opin. Virol. 32, 9-14 (2018)

33. Ding, S. W., Han, Q., Wang, J. \& Li, W. X. Antiviral RNA interference in mammals. Curr. Opin. Immunol. 54, 109-114 (2018).

34. Cullen, B. R. RNA interference in mammals: the virus strikes back. Immunity 46, 970-972 (2017).

35. Backes, S. et al. The mammalian response to virus infection is independent of small RNA silencing. Cell Rep. 8, 114-125 (2014).

36. Bogerd, H. P., Whisnant, A. W., Kennedy, E. M., Flores, O. \& Cullen, B. R. Derivation and characterization of Dicer- and microRNA-deficient human cells. RNA 20, 923-937 (2014)

37. Parameswaran, P. et al. Six RNA viruses and forty-one hosts: viral small RNAs and modulation of small RNA repertoires in vertebrate and invertebrate systems. PLoS Pathog. 6, e1000764 (2010).

38. Girardi, E., Chane-Woon-Ming, B., Messmer, M., Kaukinen, P. \& Pfeffer, S. Identification of RNase L-dependent, $3^{\prime}$-end-modified, viral small RNAs in Sindbis virusinfected mammalian cells. mBio 4, e00698-13 (2013).

39. Kennedy, E. M. et al. Production of functional small interfering RNAs by an aminoterminal deletion mutant of human Dicer. Proc. Natl Acad. Sci. USA 112, E6945-E6954 (2015). 
40. Bogerd, H. P. et al. Replication of many human viruses is refractory to inhibition by endogenous cellular microRNAs. J. Virol. 88, 8065-8076 (2014).

41. Ding, S. W. RNA-based antiviral immunity. Nat. Rev. Immunol. 10, 632-644 (2010).

42. Saldana, M. A. et al. Zika virus alters the microRNA expression profile and elicits an RNAi response in Aedes aegypti mosquitoes. PLoS Negl. Trop. Dis. 11, e0005760 (2017).

43. Varjak, M. et al. Characterization of the Zika virus induced small RNA response in Aedes aegypti cells. PLoS Negl. Trop. Dis. 11, e0006010 (2017).

44. Kozak, R. A. et al. MicroRNA and mRNA dysregulation in astrocytes infected with Zika virus. Viruses 9, E297 (2017). pii.

45. Li, H., Li, W. X. \& Ding, S. W. Induction and suppression of RNA silencing by an animal virus. Science 296, 1319-1321 (2002).

46. Sullivan, C. S. \& Ganem, D. A virus-encoded inhibitor that blocks RNA interference in mammalian cells. J. Virol. 79, 7371-7379 (2005).

47. Shan, G. et al. A small molecule enhances RNA interference and promotes microRNA processing. Nat. Biotechnol. 26, 933-940 (2008).

48. Zhang, Q., Zhang, C. \& Xi, Z. Enhancement of RNAi by a small molecule antibiotic enoxacin. Cell Res. 18, 1077-1079 (2008)

49. Abell, N. S., Mercado, M., Caneque, T., Rodriguez, R. \& Xhemalce, B. Click quantitative mass spectrometry identifies PIWIL3 as a mechanistic target of RNA interference activator enoxacin in cancer cells. J. Am. Chem. Soc. 139, 1400-1403 (2017).

50. Guryanova, O.A. et al. Nonreceptor tyrosine kinase BMX maintains self-renewal and tumorigenic potential of glioblastoma stem cells by activating STAT3. Cancer Cell 19, 498-511 (2011).

51. Li, C. et al. 25-Hydroxycholesterol protects host against Zika virus infection and its associated microcephaly in a mouse model. Immunity 46, 446-456 (2017).

52. Tsai, K., Courtney, D. G., Kennedy, E. M. \& Cullen, B. R. Influenza A virus-derived siRNAs increase in the absence of NS1 yet fail to inhibit virus replication. RNA 24, 1172-1182 (2018).
53. Samuel, G. H., Wiley, M. R., Badawi, A., Adelman, Z. N. \& Myles, K. M. Yellow fever virus capsid protein is a potent suppressor of RNA silencing that binds doublestranded RNA. Proc. Natl Acad. Sci. USA 113, 13863-13868 (2016).

54. Schnettler, E. et al. Noncoding flavivirus RNA displays RNA interference suppressor activity in insect and mammalian cells. J. Virol. 86, 13486-13500 (2012).

55. Scaturro, P. et al. An orthogonal proteomic survey uncovers novel Zika virus host factors. Nature 561, 253-257 (2018).

56. Bond, A. M., Ming, G. L. \& Song, H. Adult mammalian neural stem cells and neurogenesis: five decades later. Cell. Stem. Cell. 17, 385-395 (2015).

57. Wu, X. et al. Intrinsic immunity shapes viral resistance of stem cells. Cell 172 423-438 (2018).

58. Seo, G. J. et al. Reciprocal inhibition between intracellular antiviral signaling and the RNAi machinery in mammalian cells. Cell Host Microbe 14, 435-445 (2013).

59. Maillard, P. V. et al. Inactivation of the type I interferon pathway reveals long double-stranded RNA-mediated RNA interference in mammalian cells. EMBO J. 35, 2505-2518 (2016).

60. Li, Y. et al. Induction and suppression of antiviral RNA interference by influenza A virus in mammalian cells. Nat. Microbiol 2, 16250 (2016).

61. Hu, B. Y. \& Zhang, S. C. Differentiation of spinal motor neurons from pluripotent human stem cells. Nat. Protoc. 4, 1295-1304 (2009).

62. Li, X. F. et al. Characterization of a 2016 clinical isolate of Zika virus in non-human primates. EBioMedicine 12, 170-177 (2016).

63. Deng, Y. Q. et al. Isolation, identification and genomic characterization of the Asian lineage Zika virus imported to China. Sci. China Life Sci. 59, 428-430 (2016).

64. Shan, C. et al. An infectious CDNA clone of Zika virus to study viral virulence, mosquito transmission, and antiviral inhibitors. Cell Host Microbe 19, 891-900 (2016).

65. Wen, D. et al. N-glycosylation of viral E protein is the determinant for vector midgut invasion by flaviviruses. mBio 9, e00046-18 (2018). pii.

66. Zhang, B. et al. Differential antiviral immunity to Japanese encephalitis virus in developing cortical organoids. Cell Death Dis. 9, 719 (2018). 